\title{
"La asistencia al enfermo mental" de Luis Valenciano: la profesionalización del cuidado al enfermo mental durante la Segunda República Española
}

\section{"La asistencia al enfermo mental" of Luis Valenciano: the professio- nalization of care to mentally ill during the Second Spanish Republic "La asistencia al enfermo mental" of Luis Valenciano: a profissionalização do cuidado a ao doente mental ao longo da Segunda República Espanhola}

\author{
Alicia Duro Sánchez ${ }^{1}$, Olga Villasante ${ }^{2}$ \\ ${ }^{1}$ Graduada Universitaria en Enfermería por la Universidad Complutense de Madrid, Especialista en Salud Mental. Hospital \\ Universitario Severo Ochoa (Leganés). \\ ${ }^{2}$ Psiquiatra. Hospital Universitario Severo Ochoa (Leganés).. \\ Cómo citar este artículo en edición digital: Duro Sánchez, A. y Villasante, O. (2016). "La asistencia al enfermo mental" \\ de Luis Valenciano: la profesionalización del cuidado al enfermo mental durante la Segunda República Española. \\ Cultura de los Cuidados (Edición digital), 20, 44. Disponible en:< http://dx.doi.org/10.14198/cuid.2016.44.05> \\ Correspondencia: Hospital Universitario Severo Ochoa. Av. Orellana, s/n, 28911, Leganés (Madrid) \\ Correo electrónico: ovillasante.hsvo@salud.madrid.org \\ Recibido: 11/06//2015; Aceptado: 03/10/2015
}

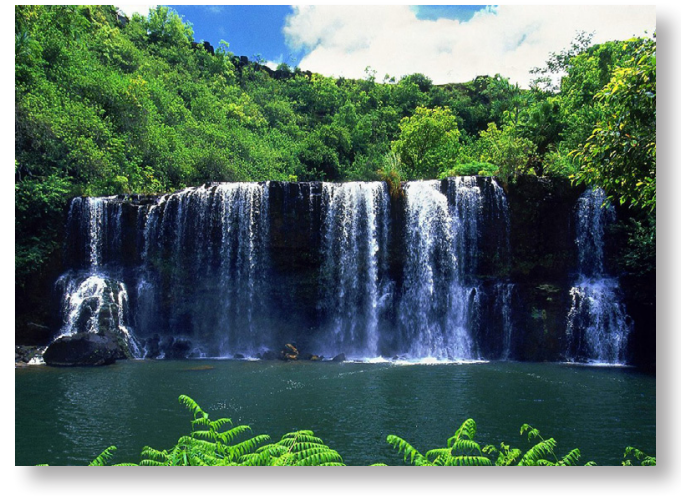

ABSTRACT

The reform of psychiatric care, driven by legislative changes implemented throughout the Second Spanish Republic, proposed improvements in the professionalization of caregivers of psychiatric facilities. By Decree of May 16, 1932 junior staff was organized in Practitioners of Medicine and Surgery diploma nurses and psychiatric nurses; and the Diploma in Psychiatric Nurse was regulated. By the Decree of May 17, 1932 the official curriculum was published for this degree and the
July 5, 1932, the basis of a competition for a work that would fit the agenda. Psychiatric Superior Council elected and recommended " $\mathrm{La}$ asistencia al enfermo mental"(1933) by Luis Valenciano Gaya. The aim of this paper is to analyze the content of the book, including the care the mentally ill needed, as well as physical and attitudinal requirements for being "psychiatric nurse". It also seeks to value the importance of the manual, while it was not the first published in Spanish, truly presented a national circulation and became the reference work for psychiatric nurses in the 30s.

Keywords: Psychiatric nursing, Luis Valenciano Gaya, Second Spanish Republic, Nursing History, Mental Health.

\section{RESUMO}

A campanha de reforma da assistência psiquiátrica, impulsada a través de mudanças legislativas impostas ao longo da Segunda República Espanhola, planteou melhoras na profissionalização dos cuidadores nos estabe- 
lecimentos psiquiátricos. A través do Decreto de 16 de Maio de 1932 o pessoal subalterno foi organizado em Practicantes em Medicina e Cirurgia com diploma de enfermeiros psiquiátricos e Enfermeiros e Enfermeiras Psiquiátricos; e regulamentou-se a obtenção do Diploma de Enfermeiro Psiquiátrico. No Decreto de 17 de Maio de 1932 foi publicado o programa oficial de estudos para obter o nomeado título e, no dia 5 de Julho de 1932, as bases de um concurso para uma obra que ajustar-seá ao temário. O Conselho Superior Psiquiátrico escolheu e recomendou "La asistencia al enfermo mental" escrita por Luis Valenciano Gayá, publicada em 1933. O objetivo deste trabalho é analisar o conteúdo do livro, que incluía os cuidados que precisavam os doentes mentais, assim como as condições físicas e actitudinais requeridas para ser "doente psiquiátrico". Assim mesmo trata-se de avaliar a importância deste primeiro manual que, ainda não sendo o primeiro publicado em espanhol, apresentou uma difusão nacional e tornou-se obra de referência para os enfermeiros psiquiátricos nos anos trinta.

Palavras chave: Enfermaria psiquiátrica, Luis Valenciano Gaya, Segunda República Espanhola, História da Enfermaria, Saúde Mental.

\section{RESUMEN}

La reforma de la asistencia psiquiátrica, impulsada a través de los cambios legislativos implantados durante la Segunda República Española, planteó mejoras en la profesionalización de los cuidadores de los establecimientos psiquiátricos. A través del Decreto de 16 de mayo de 1932 se organizó el personal subalterno en Practicantes en Medicina y Cirugía con el diploma de enfermeros psiquiátricos y Enfermeros y Enfermeras Psiquiátricos; y se reguló la obtención del Diploma de Enfermero Psiquiátrico. En el Decreto de 17 de mayo de 1932 se publicó el programa oficial de estudios para obtener dicha titulación y, el 5 de Julio de 1932, las bases de un concurso para una obra que se ajustara al temario. El Consejo Superior Psiquiátrico eligió y recomendó "La asistencia al enfermo mental"(1933) de Luis Valenciano Gayá. El objetivo de este trabajo es analizar el contenido del libro, que incluía los cuidados que precisaban los enfermos mentales, así como las condiciones físicas y actitudinales requeridas para ser “enfermero psiquiátrico". Asimismo se pretende valorar la importancia del manual que, si bien no fue el primero publicado en español, si presentó una difusión nacional y se convirtió en la obra de referencia para los enfermeros psiquiátricos en los años treinta.

Palabras clave: Enfermería psiquiátrica, Luis Valenciano Gaya, Segunda República, Historia de Enfermería, Salud Mental

\section{INTRODUCCIÓN}

A dos meses de constituirse la República, el Gobierno Provisional publica el Decreto sobre la asistencia a enfermos mentales de 3 de Julio de 1931, que marcará el comienzo de la transformación de la asistencia psiquiátrica (Campos, Huertas, 1998). La finalidad principal del citado Decreto fue la regulación de la admisión y las altas de los enfermos psíquicos en los establecimientos psiquiátricos, además de los permisos temporales y las formalidades del reingreso (Gaceta 7 de Julio 1931). Se planteaba un nuevo modelo asistencial, que pretendía alejarse del encierro manicomial que se había realizado previamente, incorporando los aspectos profilácticos de la enfermedad mental (Huertas, 2003).

Las propuestas de cambio también se vieron reflejadas a través de la creación de diferentes instituciones. En el Decreto del 10 noviembre de 1931 se crea el Consejo Superior Psiquiá- 
trico, presidido por Gonzalo Rodríguez Lafora (1886-1971), con funciones asesoras de programación e inspección (Gaceta 12 de Noviembre 1931). Por otro lado, en diciembre de 1931 surge, en la Dirección General de Sanidad, la Sección Central de Psiquiatría centrada en la dirección y ejecución directa (Gaceta 2 de Diciembre 1931) y, el 16 de abril de 1932, se crea el primer Patronato de Asistencia Social Psiquiátrica encargado de la vigilancia postmanicomial de los enfermos mentales por medio de Establecimientos oficiales o particulares de la provincia de Madrid (Gaceta 17 de abril 1932).

La campaña de reformas de la asistencia psiquiátrica, iniciada durante las primeras décadas del siglo XX y previa a los cambios legislativos señalados, también había planteado cambios en la formación y organización del personal subalterno de los establecimientos psiquiátricos (Ventosa, 2000). Concretamente, en el Primer Congreso de la AEN, celebrado en junio de 1926 en Barcelona, se retomaron las peticiones de la Sociedad de Neurología $i$ Psiquiatría que recomendaban el aumento del número de técnicos, internos, enfermeras y su instrucción. También, en la cuarta Asamblea de la Liga de Higiene Mental, celebrada en Zaragoza en 1930, Eduardo Guija Morales (1904-1966) se había pronunciado sobre la formación y admisión del personal subalterno de los manicomios públicos, antesala de la regulación del Diploma de enfermero Psiquiátrico (Villasante, 2013).

\section{EL DIPLOMA DE ENFERMERO PSIQUIÁ- TRICO Y LA PROFESIONALIZACIÓN DE LOS CUIDADOS DURANTE LA SEGUN- DA REPÚBLICA}

En consonancia con el avance que experimentó la enfermería en diferentes áreas (Bernabé, Gascón, 1999; Vera Pérez, Hernández
Conesa, 2011), un cambio legislativo que marcó un hito histórico para la formación de la enfermería psiquiátrica fue la Orden Ministerial firmada por el Director General de Sanidad, Casares Quiroga (1884-1950), el 16 de mayo de 1932. No se trataba de una reglamentación aislada, sino que respondía al cambio progresivo de las estructuras sanitarias españolas que tuvo lugar durante esos años y que alcanzó su punto álgido en el período republicano (Villasante, 2013). A través de este decreto se organizó el personal subalterno y se reguló la obtención del Diploma de Enfermero Psiquiátrico (Herrera, 1990). Se dividió el personal sanitario subalterno de los establecimientos psiquiátricos, públicos y privados, en Practicantes en Medicina y Cirugía con el diploma de enfermeros psiquiátricos y Enfermeros y Enfermeras Psiquiátricos. Los practicantes se encargarían de "asegurar la práctica médica y quirúrgica de urgencia, diurna y auxiliar a los médicos que tengan que prestar asistencia en los Establecimientos" y se establecía dos practicantes para cincuenta pacientes. Los enfermeros psiquiátricos realizarían el cuidado directo, siendo "el elemento auxiliar principal de la asistencia psiquiátrica intramanicomial'. La proporción, en este caso, sería de uno por diez enfermos que precisaran vigilancia continua y de uno por 150 de pacientes en vigilancia discontinua (Gaceta 20 de Mayo 1932).

A través del citado Decreto de 16 de mayo de 1932 también se planteó, en relación a la función de los Enfermeros Psiquiátricos, que "la importancia de su misión exige una base de conocimientos técnicos y prácticos". Se establecieron los requisitos para la obtención del Diploma de Enfermero Psiquiátrico y se dispuso la celebración de un examen anual en la fecha fijada por la Dirección General de Sanidad, en los distritos universitarios de Madrid, Barce- 
lona, Granada y Santiago. Dos meses después, el 19 de julio de 1932 se aceptó la solicitud del director y subdirector del Manicomio Provincial de Valencia de incluir también el distrito universitario de dicha ciudad para la realización de los exámenes (Gaceta 28 de Julio 1932).

En el Decreto de 17 de mayo de 1932 se publicó el programa oficial de estudios para obtener el certificado de aptitud de "enfermero psiquiátrico", que constaba de 24 lecciones, supervisadas por el Consejo Psiquiátrico (Gaceta 20 de Mayo 1932). El contenido incluía nociones de psicopatología y cuidados del enfermo mental, pero además varios temas sobre cuidados generales y nociones de patología general, ya que no hay que olvidar que el personal de cuidado de las instituciones psiquiátricas, en muchas ocasiones, presentaban escasa o nula formación sanitaria. De hecho, los problemas con el personal subalterno de las instituciones psiquiátricas ya habían sido identificados, entre otros por Gonzalo Rodríguez Lafora, que había afirmado: "los enfermeros eran nombrados por una junta clasificadora no médica $y$ ajena al funcionamiento del hospital, distribuyendo los cargos entre licenciados del Ejército e individuos cuya conducta y carácter era cuestionable. Dada su independencia de la dirección médica no obedecían las órdenes de vigilancia o de tratamiento dadas por los facultativos, maltratando y abandonando a los enfermos y negándose a todo estudio de formación especial" (Rodríguez Lafora, 1933). De hecho, una de las diferencias de los enfermeros con médicos y practicantes era que los primeros no pertenecían al cuerpo de beneficencia y Rodríguez Lafora denunció que los directores de los establecimientos, dependientes del Estado, debían aceptar un elevado porcentaje de licenciados del Ejercito, quienes considerándose seguros en sus puestos por la protección de la ley mi- litarista, no se veían obligados a respetar a sus superiores jerárquicos.

Los enfermeros no contaban, tampoco con textos que incluyeran un contenido formativo amplio, ya que los libros de enfermería publicados hasta entonces, dedicaban pocas páginas al cuidado de los enfermos mentales, como puede comprobarse en el Manual del auxiliar de medicina y cirugía de Felipe Sainz de Cenzano (1934), cuya primera edición data de 1907 o Las Carreras Auxiliares Médicas (Emilio Alonso y García Sierra, 1923). Por otra parte, el único manual específico, publicado, hasta ese momento, sobre los cuidados psiquiátricos era el Manual del Enfermero en los Manicomios (1909), escrito por Vicente Goyanes Cedrón en el Manicomio de Conxo, prácticamente sin difusión fuera del ámbito local (Villasante, 2015). Por ello el 5 de Julio de 1932 se publicaron las bases de un concurso entre "médicos españoles" para la presentación de una obra que se ajustara al programa del 20 de mayo de 1932, para la obtención del Diploma de Enfermero Psiquiátrico (Gaceta 5 de Julio 1932).

Se requería que las obras se presentaran antes del 31 de Diciembre de ese mismo año, en cuartillas, escritas a máquina por un solo lado y en sobre cerrado, lacrado por el sistema de lema y plica. La obra premiada, no sería considerada "texto oficial u obligado" sino "recomendado" y plantearon que, cada tres años, se repetiría el concurso, para que el libro estuviera actualizado y adecuado a la asistencia psiquiátrica del momento. El Consejo Superior Psiquiátrico recibió cuatro manuales, bajo los lemas "Ciencia y Conciencia", "Simón”, “Ciencia y Paciencia” y "Mateo Alemán”, que, tras ser estudiados y revisados, se eligió la obra bajo el lema "Simón" escrita por Luis Valenciano (Gaceta 14 de Julio 1933) y publicada como "La asistencia al enfermo mental". 
"LA ASISTENCIA AL ENFERMO MENTAL”: UN MANUAL PARA ENFERMEROS RECOMENDADO POR EL CONSEJO SUPERIOR PSIQUIÁTRICO (Imagen I).

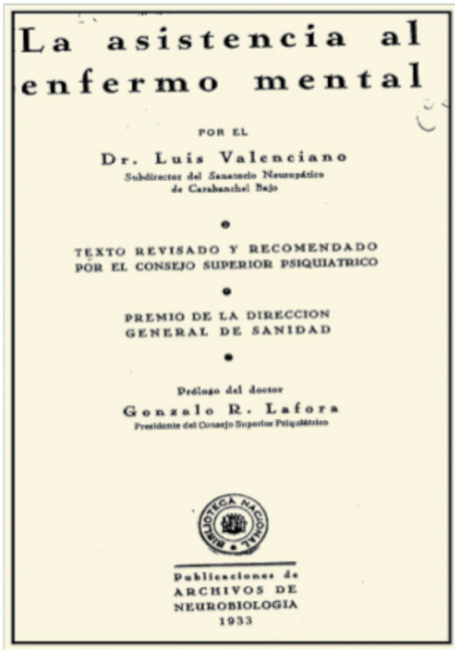

Imagen I: Portada del Manual

Este primer texto, orientado a la profesionalización de la enfermería psiquiátrica, fue escrito por Luis Valenciano Gayá, psiquiatra formado junto a Rodríguez Lafora en el Hospital Provincial, y publicado en 1933, dentro de las Publicaciones de Archivos de Neurobiología. La extensión alcanza las 357 páginas - estructuradas en dos partes, agrupadas a su vez en 3 apartados-, una diferencia significativa con El manual de los enfermeros de los manicomios que, como hemos mencionado, era el único texto previo dirigido específicamente a los cuidadores del enfermo mental, que no superaba las 30 páginas. La obra comienza con un prefacio elaborado por Rodríguez Lafora, el 2 de noviembre de 1933, donde se pone de manifiesto la necesidad de una especialización del personal subalterno psiquiátrico y el objetivo principal de la obra se expresa del siguiente modo: "modificar radicalmente el trato inadecuado que hasta entonces se daba en nuestro país a los enfermos mentales" (Valenciano:5). Si bien más de un siglo antes, concretamente en el artículo 122 de la orden de Beneficencia de 1822 publicada durante el Trienio Liberal se habían prohibido explícitamente los malos tratos (Villasante 2005). La realidad es que el cuidado de los enfermos estaba en manos de los "loqueros", nombre con el se conocían popularmente. La descripción de los cuidadores que realizó Benito Pérez Galdós (1843-1920) en La desheredada (1881), cuando la protagonista de esta novela realista, Isidora Rufete, va a visitar a su padre al Manicomio de Leganés es realmente desgarradora: Dos loqueros, membrudos, aburridos de su oficio, se pasean atentos, como polizontes que espían el crimen... No hay compasión en sus rostros, ni blandura en sus manos, ni caridad en sus almas. De cuantos funcionarios ha podido inventar la tutela del Estado, ninguno es tan antipático, como el domador de locos. Carcelero-enfermero, es una máquina muscular que ha de constreñir en sus brazos de hierro al rebelde y furioso" (Pérez Galdós, 1881, p. 19). Independientemente de las licencias literarias que el autor pudo haberse tomado en la novela, la atención a los pacientes estaba escasamente profesionalizada y, de hecho, en 1916, Lafora denunció la situación de los enfermos mentales en su artículo "Los manicomios españoles" publicado en la revista España (Rodríguez Lafora, 1916). Lo novedoso de este artículo impactante fue, además de la denuncia sobre el departamento de alineados del Hospital Provincial de Madrid, la publicación de fotografías de pacientes mentales encadenados, desnudos sobre lechos de paja y cubiertos con mantas, ingresados en el Manicomio Provincial de Granada y el Manicomio Provincial de Valencia (Villasante, 2011).

El libro de Valenciano, en las palabras preliminares del propio autor, indica que el contenido 
se basa en los cursillos para enfermeros impartidos en el Sanatorio Neuropático de Carabanchel (Huertas, 2002). En Madrid, además de los cursos en este sanatorio privado propiedad de Rodríguez Lafora, el 18 de septiembre de 1933 se comenzó un curso en el Hospital General para practicantes, enfermeros y enfermeras psiquiátricos, cuya matrícula se había abierto en agosto, previo anuncio en el $A B C(A B C, 4$ de agosto 1933, p.28). Las conferencias de carácter teórico fueron impartidas, en días alternos por las tardes en horario de 7 a 8 en el Hospital de la Beneficencia General por Mendiguchía Carriche, Fernández Méndez, Martín Vegué y Moreno Rubio y la parte práctica en días festivos, de once a una, en el Manicomio de Leganés, donde trabajaban los citados médicos (Tierno, Villasante, Vázquez de la Torre, 2007). La conferencia de clausura del curso corrió a cargo de Fernández Sanz, entonces, jefe facultativo del Manicomio Nacional, y que formaba parte de ese grupo de psiquiatras (Huertas, 2002) que ejerció gran influencia en la reforma psiquiátrica ya que había sido presidente de la Liga de Higiene Mental (1927), Inspector de Manicomios Nacionales (1930) y Vicepresidente del Consejo Superior Psiquiátrico (1931) (Candela, Villasante, 2011).

Desconocemos si en todas las instituciones psiquiátricas españolas se organizaron cursos de formación, pero, sin duda, podemos afirmar que la Orden ministerial del 16 de mayo de 1932 fue un detonante para el inicio de actividades formativas enfocadas a los cuidadores de enfermos mentales y para la publicación de diversos libros. Además de las obras previamente mencionadas, se publicaron, entre otras, las Lecciones teóricas y prácticas para contestar al curso elemental de enfermeros psiquiátricos (1935), del subdirector del Sanatorio de Ciempozuelos, Eulogio García de la Piñera, el Manual de la enfermera general y psiquiátrica de José Salas
(1935), médico que trabajó en el manicomio de Mujeres de Ciempozuelos o, en 1936, el Prontuario del enfermero psiquiátrico de Francisco Domingo Simó, director del Manicomio de Valencia, no obstante aludían al libro de Valenciano como manual de referencia.

En la región catalana, abanderada en el movimiento para formar una enfermería especializada, hay que destacar al psiquiatra Óscar Torras, uno de los comisionados para discutir los términos de la formación de enfermeros psiquiátricos (Comelles, 2005). Belarmino Rodríguez Arias (1895-1997), profesor de Neurología de Medicina de la Universidad Autónoma de Barcelona y Presidente de la Comisión asesora psiquiátrica de la Generalitat de $\mathrm{Ca}$ talunya dirigió a los médicos del Manicomio de S. Baudilio de Llobregat (Barcelona) para la publicación del Manual del enfermero para pacientes nerviosos y mentales, cuya traducción corrió a cargo de F. Capó Balle y E. Irazoqui Villalonga. Este texto de los profesores suizos W. Morgenthaler, y O. L. Forel (Morgenthaler, Forel, 1936), ya editado en alemán, francés e italiano para cubrir las variaciones idiomáticas de Suiza (Herrera, 2000), fue citado por Valenciano en la bibliografía del texto para enfermeros. Si bien esta traducción del manual de Morgenthaler tuvo gran difusión en España, el libro de Valenciano fue, sin duda, el más reconocido e influyente de los textos españoles en los años treinta y, si bien, aún existe una laguna historiográfica en este área, seguramente también se mantuvo como libro de referencia durante las primeras décadas de la posguerra (Villasante, en prensa).

\section{LA INFLUENCIA EUROPEA EN LA OBRA DE LUIS VALENCIANO}

El manual de Valenciano, dividido en dos partes, incluía los contenidos necesarios para 
formar al personal subalterno y permitir la obtención del diploma de enfermero psiquiátrico en un libro de "fácil comprensión y de carácter práctico" (Valenciano:9). En la primera parte se exponen los "Fines de la Asistencia Psiquiátrica”, las "Condiciones del Enfermero" y "La Asistencia al Enfermo Psíquico" y, en la segunda, se incluía un extenso apartado sobre las “Nociones de Psicología y Psiquiatría”, ya que no se puede olvidar que gran parte de los cuidadores tenían una escasa instrucción general $y$, por ende, sanitaria. La formación en psicopatología comenzaba con una formación básica sobre Anatomía y Fisiología, continuando con la definición de conceptos psicológicos y finalizaba con un amplio recorrido sobre las diferentes enfermedades mentales y unas Nociones de Psicoterapia. Las enfermedades psiquiátricas se organizaban en los 14 grupos correspondientes a las categorías nosográficas de Emil Kraepelin, ya que el 28 de noviembre de 1931 se había ordenado a todos los establecimientos psiquiátricos clasificaran a los enfermos mentales de acuerdo a los criterios Kraepelianos (Gaceta 2 de Diciembre 1931). No obstante, es reseñable que, en el apartado "La asistencia especial a los distintos tipos de enfermos psíquicos" (Valenciano:125), los enfermos no se agrupaban por criterios nosográficos, sino en relación a la conducta $y$, por tanto, en base a los cuidados que precisaban: agresivos y agitados, toxicómanos, enfermos con ideas de suicidio, enfermos socialmente peligrosos, anormales sexuales, enfermos que rehusan la alimentación, negativistas, aislados $y$ ensimismados, enfermos que presentan alucinaciones e ideas delirantes, destructores, enfermos que padecen ataques, sucios, desordenados e impedidos.

Cuando el libro de Valenciano se editó habían pasado casi cinco décadas del Handb- book for the instruction of attendants on the insane, publicado por primera vez en 1885 por la Médico-Psychologique Association (Nolan, 1993). El psiquiatra español citó en la Bibliografía la edición de 1931, denominada Handbook for mental nurses, junto al ya referido de Morgenthaler y Forel, el Manuel technique de l'infirmier des Etablissements d'alienes de Mignot y Marchand (1931), cuya primera edición había sido publicada en 1912 y el texto Der seelisch kranke Mensch und seine Pflege [El enfermo mental y su cuidado] de 1925, obra finlandesa traducida al alemán por su propia autora, Neuman-Rahn, entre otras publicaciones. Durante su formación en Berlín, Viena, Múnich y Zúrich, Valenciano había conocido estos libros que habían sido impulsados por Sociedades médico-psicológicas y, en ocasiones, por "asociaciones de auxilio a pacientes mentales", agrupaciones prácticamente desconocidas en España. De ese modo el psiquiatra murciano no tuvo pretensiones de originalidad y afirmó que "hubiera sido injusto y aventurado desdeñar la abundante literatura que sobre este punto existe en países que han abordado con anterioridad a nosotros el problema de la formación de los enfermeros psiquiátricos, con tan excelentes resultados" (Valenciano:9).

Así pues, gran parte de los contenidos son similares a los libros europeos, a los que se añadió un Apéndice final sobre la Legislación se incluía, además, del Decreto sobre la asistencia de los enfermos mentales de 3 de julio de 1931 (Campos, Huertas, 1998), y el Decreto de 20 de mayo de 1932 por el que se había creado el Diploma de Enfermero Psiquiátrico y el artículo 575 del Código Penal, que castigaba a los encargados de la custodia que dejaran vagar por las calles a los enfermos mentales (Valenciano:325-338). La obra concluye con un Índice alfabético facilitando la localización 
de términos utilizados en la obra y un Índice de materias, donde se plasma el índice general y las referencias de 36 ilustraciones. Entre estas últimas se incluían esquemas, dibujos de anatomía, técnicas de enfermería, fotografías de centros psiquiátricos europeos y alguna del Hospital General de Madrid, donde Valenciano trabajó (Imagen II)

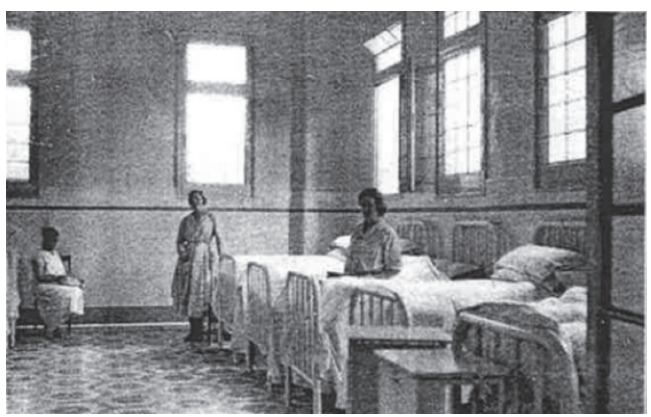

Imagen II: Antesala de una sala de guardia (Departamento psiquiátrico del Hospital General de Madrid)

\section{LAS CONDICIONES DEL ENFERMERO PSIQUIÁTRICO: INSTRUCCIÓN, MORA- LIDAD Y SUBORDINACIÓN}

Una parte significativa del texto " $L a$ asistencia al enfermo mental" se dedica a las condiciones físicas y actitudinales requeridas al enfermero psiquiátrico y la visitadora psiquiátrica; ésta última era una categoría para la que se requería un año de experiencia como enfermera psiquiátrica (Siles, 1996). Las condiciones físicas consistían en una buena presencia y salud, que incluía fortaleza, destreza, carencia de defectos físicos, y buen funcionamiento de los sentidos, además de una buena instrucción escolar. En referencia a la actitud del enfermero psiquiátrico, se valoraba la discreción, la honradez, moralidad y la energía necesaria para hacer cumplir las pautas médicas, con dominio de sí mismo. También se destacaba la importancia del respeto hacia el enfermo mental, el interés y la capacidad de trabajo, empatía y compenetración con los sufrimientos de estos, a lo que había que añadir vocación, firmeza, buenas dotes sociales y respeto hacia el secreto profesional, además de gran conocimiento del medio en el que vivía el paciente, en el caso de las visitadoras.

Sin embargo, en España no se contaban con los instrumentos para medir estas excepcionales cualidades requeridas, según Valenciano, a enfermeros y enfermeras. Es cierto que, en las primeras décadas del siglo XX, las corrientes sobre la caracterología y el temperamento se habían concretado en investigaciones e instituciones dedicadas a la orientación laboral y habían destacado los trabajos del Instituto de Orientación Profesional de Barcelona y, posteriormente, el Institut Psicotècnic y el Instituto de Psicotécnico de Madrid, bajo la presidencia de José Germain. La valoración psicológica aplicada a diferentes profesiones también había llegado al personal sanitario, cuestión en la que Emili Mira había sido pionero en nuestro país al plantear un test para determinar la capacidad de discriminación ética del personal médico auxiliar, si bien no fueron aplicadas de una forma generalizada en las instituciones psiquiátricas. El psicólogo catalán conocía la investigación de Fritz Giese desarrolladas en Halle, que pasaban por ser los primeros trabajos psicotécnicos acerca de la selección del personal auxiliar de los sanatorios. Por otra parte, Rodríguez Lafora había pronunciado una conferencia en el Instituto de Psicotécnico de Madrid en abril de 1932, sobre la selección de enfermeros psiquiátricos. En un artículo contemporáneo el presidente del Consejo Superior y promotor del Diploma de enfermero psiquiátrico había destacado que la selección en España era prácticamente imposible por la presencia de los trabajadores del Ejército, la incultura del personal subalterno y la implantación brusca de la jornada de 8 horas que impedía preparar al personal idóneo para la 
responsabilidad del cuidado (Rodríguez Lafora, 1933).

Por otra parte la autonomía de la enfermería estaba aún lejos de considerarse y el papel del Enfermero psiquiátrico se consideraba un auxiliar médico y un cuidador, frente a un perfil más técnico del Practicante. De ese modo los enfermeros debían responder con una " $a b$ soluta subordinación" ante las órdenes del facultativo, cumpliéndolas aunque no estuvieran conformes, idea que se repite, en varias ocasiones en el libro de Valenciano. Además éste debía contribuir a reducir los prejuicios y miedos que existían entre la población general, cuidar la reputación del establecimiento psiquiátrico mediante un comportamiento adecuado en el exterior y el respeto de los datos confidenciales de los pacientes.

Es especialmente interesante, a nuestro modo de entender, la capacidad de observación y destreza exigida al enfermero psiquiátrico, que José Salas expresó en los siguientes términos: «la enfermera psiquiátrica ha de proporcionar al facultativo los datos de observación que éste no pueda apreciar de un modo directo. Se fijará tanto en los síntomas psíquicos como en los somáticos» (Salas, 1935, p. 293). En un momento en el que los registros escritos del personal auxiliar no estaban aún estandarizados en las instituciones psiquiátricas, cuestión que merecería comprobar con estudios de caso, se insistía en escribir en hojas especiales o cuadernillos, para evitar los olvidos y equivocaciones. De ese modo, los médicos podrían comprobar cuestiones como la temperatura, el pulso, las respiraciones, la micción y defecación, así como los "vicios sexuales" u otros comportamientos de los pacientes. A juicio de Valenciano, en cada departamento del centro, tenían que emplearse las hojas de registro que la dirección decidiera, entre las que enumera- ba: el cuaderno de visita, el de medicamentos, el de observaciones cotidianas, las hojas de temperatura y peso, las hojas de ataques epilépticos, la lista de enfermos bañados, de trabajadores, de ropa interior y de trajes de los enfermos, la relación diaria de enfermos existentes en el departamento, de camas libres y la relación de medios de contención mecánica empleados y de inyecciones calmantes aplicadas por los médicos (Valenciano:319). A modo de ejemplo, en el libro de Valenciano se reproducen seis pliegos procedentes otros establecimientos psiquiátricos, como los cuestionarios I (valoración al ingreso), II (datos que tiene que aportar el médico al ingreso) y III (se debería rellenar después de unos días de ingreso y periódicamente -tres meses, seis meses, un año...). Estos cuestionarios pertenecían al libro de Morgenthaler "Die Pflege der Gemütsund Nervenkranken" (Manual de enfermero para pacientes nerviosos y mentales) de 1930, mientras que las "Notas de la Enfermera" y el impreso "Variaciones cotidianas del enfermo" pertenecían a la Casa de Salud de Valdecilla (Valenciano: 68-73).

Además de esta observación activa, los enfermeros debían implicarse en la labor terapéutica específicamente psiquiátrica (Miqueo, Muñoz, 2014), que incluía los cuidados físicos (medidas higiénico-dietéticas), mecánicos y químicos, además de las técnicas médicoquirúrgicas. De hecho, la psiquiatría de corte asilar hasta entonces había introducido, en los años previos, innovaciones terapéuticas como las técnicas de sueño prolongado de Jakob Kläsi, la paludoterapia de Julius Wagner von Jauregg y otros métodos de piretoterapia, etc. Valenciano reprodujo la "Hoja para la cura de Kläsi” (Valenciano:74), tomada del Establecimiento psiquiátrico de Burghölzli, de Zúrich, un cuadro en el que debían anotarse los hip- 
nóticos, la temperatura, las características del sueño prolongado, etc.

Es también relevante el planteamiento que se realiza sobre la higiene mental, que abarcaba la prevención de la enfermedad mental, desde áreas como la escuela y el ámbito laboral. El psiquiatra abordó un aspecto polémico de la prevención que consistía en la esterilización de los enfermos mentales como medida preventiva, apoyándose en los estudios que demostraban la herencia genética de la enfermedad mental, "con ello la raza obtiene un beneficio y el propio individuo también, ya que si su estado mental se lo permite puede casarse libremente" (Valenciano:321). Valenciano justifica esta medida preventiva en que ya estaba legalmente permitida en Norteamérica y en Europa, concretamente en Suiza y Alemania. De hecho las ideas eugénicas habían cobrado gran auge en nuestro país durante las primeras décadas del siglo XX, siendo Tomás Busquet Teixidor uno de los grandes divulgadores de esta corriente, consideraba que la higiene mental tiene dos finalidades: "una puramente humanitaria y otra francamente egoística, de protección y defensa de la sociedad y de la raza" (citado en Álvarez Peláez, 2003, p. 117).

\section{A MODO DE EPÍlOGO}

Es interesante señalar que la formación en España durante las primeras décadas del siglo XX fue impulsada por los facultativos que trabajaban en las principales instituciones psiquiátricas del país. Si bien tampoco los neuropsiquiatras tenían reconocida oficialmente la especialidad, llevaban ya algunas décadas intentando legitimarse como especialistas y percibieron la escasez de la formación del personal subalterno que cuidaba a los enfermos mentales. A partir de la publicación, el 20 de mayo de 1932, del programa oficial para la obtención del diploma de Enfermero Psiquiátrico se comenzó a incrementar el número de publicaciones orientadas a la mejora formativa del personal subalterno de las instituciones psiquiátricas. Si bien, el manual de Luis Valenciano no se puede considerar, en sentido estricto, el primer libro para la enfermería psiquiátrica español, es, sin duda, el primero de carácter nacional, recomendado por el Consejo Superior, cuestión que determinó que fuese considerado un manual de referencia y el libro más relevante e influyente de la época para la formación de la enfermería psiquiátrica.

A pesar de la publicación de diferentes libros y manuales, la falta de estructuras asociativas (Segura, Hernández, Beneit, 2012) y la ausencia de un órgano de expresión (Nebreda, 2011), entre otros factores, son algunas de las causas que retrasarán la conformación de la especialidad dentro de la enfermería psiquiátrica en España. La guerra interrumpió este proceso y la introducción del personal más formado en las instituciones psiquiátricas ha sido aún un proceso tortuoso y lento que se corresponde con esta escasa especialización de la enfermería y merece, no obstante, un estudio más detallado.

\section{FUENTES}

- ABC, 4 de agosto 1933, p.28.

- Alonso García-Sierra E. (1923). Las Carreras Auxiliares Médicas. Madrid: Librería Internacional de Romo.

- Doming Simó F. (1936). Prontuario del enfermero psiquiátrico. Valencia: Imp. V. Climent Vila.

- Gaceta de Madrid, 7 de Julio de 1931, 188, 186-189.

- Gaceta de Madrid, 12 de Noviembre de 1931, 316, 932933.

- Gaceta de Madrid 2 de Diciembre de 1931, 336, 13761377.

- Gaceta de Madrid, 17 de Abril de 1932, 108, 408.

- Gaceta de Madrid, 20 de Mayo de 1932, 141,1334-1335. 
- Gaceta de Madrid, 5 de Julio de 1932, 187, 128.

- Gaceta de Madrid, 28 de Julio de 1932, 210, 733-734.

- García de la Piñera E. (1935). Lecciones teóricas y prácticas para contestar al curso elemental de enfermeros psiquiátricos. Ciempozuelos: Imprenta hermanos de San Juan de Dios.

- Goyanes Cedrón, V. (1909). Manual del enfermero en los Manicomios. Santiago: Imp. de José Ma Paredes. Este texto se halla reproducido en Revista de la Asociación Española de Neuropsiquiatría, 2015; 35(126),409-421.

- Mignot R, Marchand L. (1931) Manuel technique de l'infirmier des Etablissements d'alienes.Paris : G. Doin et Cie.

- Morgenthaler W, Forel O L. (1936) Manual del enfermero para pacientes nerviosos y mentales. Barcelona: Salvat Editores.

- Pérez Galdós, B. [1881] (1976). La desheredada. Madrid: Alianza editorial.

- Royal Medico-psychological Association (1885). Handbook for the instruction of attendants of the insane.1st ed. London: Baillière, Tindall \& Cox, consultado el 20 de junio de 2015 en http://archive.org/details/ handbookforinst01assogoog.

- Rodríguez Lafora, G. (1916). Los manicomios españoles. España; 90:8-10.

- Rodríguez Lafora G. (1933). Selección profesional de enfermeros psiquiátricos y distribución de trabajo. Archivos de Neurobiología,343-68.

- Salas, J. (1935). Manual de la enfermera general y psiquiátrica. Madrid: Hermanas Hospitalarias del Sagrado Corazón de Jesús.

- Valenciano, L. (1933). La asistencia al enfermo mental. Madrid: Publicaciones de Archivos de Neurobiología.

\section{BIBLIOGRAFÍA}

- Álvarez Nebreda CC. (2011). Bases para la publicación de una revista nacional (1934). En: Álvarez Nebreda CC. Semblanzas de la profesión de enfermera (1862-1953) (pp. 651-5). Madrid: Colegio Oficial de Enfermería de Madrid.

- Álvarez Peláez R. (2003). Higiene mental y eugenesia. Frenia, 3(1), 115-47.
- Bernabé Mestre, J.,Gascón Pérez, E. (1999). Historia de la Enfermería de Salud Pública en España (18601977). Alicante: Publicaciones de la Universidad de Alicante. Disponible en: http://rua.ua.es/dspace/bitstream/10045/14595/1/Bernabe u_Gascon_Historia_enfermeria.pdf . [Consultado el 13/06/2015].

- Candela, R., Villasante, O. (2011). Las Historias Clínicas del Manicomio de Leganés (1924-1931): Enrique Fernández Sanz y la Nosología Kraepeliana. En Del Plesistoceno a nuestros días. Contribuciones a la Historia de la Psiquiatría (pp. 212). Madrid: Asociación Española de Neuropsiquiatría.

- Campos, R., Huertas, R.(1998). Estado y asistencia psiquiátrica en España durante el primer tercio del siglo XX. Revista de la Asociación Española de Neuropsiquiatría, 18(65), 99-108.

- Comelles J. M.(2005). El purgatorio del Doctor Torras. Reforma y reacción en la psiquiatría catalana del XX. Frenia; 5, 101-132.

- Herrera Rodríguez, F. (1990). La titulación del enfermero psiquiátrico en la II República española. Minutos Mena$\operatorname{rini}(\mathrm{Ba}), 167,4-8$

- Herrera Rodríguez F. (2000). Un manual de enfermería, Híades. Revista de Historia de la Enfermería, 7, 127-132.

- Huertas, R. (2002). Los médicos de la mente. De la neurología al psicoanálisis (Lafora, Vallejo-Nágera, Garma). Madrid: Nivela.

- Huertas, R. (2003). El debate sobre la reforma psiquiátrica en la España del primer tercio del siglo XXI. Átopos, 1, 43-53.

- Miqueo C, Muñoz B. (2014). La enfermería Psiquiátrica. En Historia de la Psiquiatría del siglo XX en Aragón (pp. 337-369). Zaragoza: Sociedad Aragonesa y Riojana de Psiquiatría.

- Nolan PW. (1993). A history of the training of asylum nurses. Journal of Advanced Nursing, 18(8):1193-1201.

- Siles González J, García Hernández E. (1996). Origen histórico de la profesionalización de los cuidados mentales: los practicantes, enfermeros y visitadoras psiquiátricas. Enfermería Científica, 174-5, 49-53.

- Tierno R, Villasante O and Vázquez de la Torre P (2007). El Manicomio Nacional de Leganés: entre la reforma le- 


\section{Cultura de las Cuidados}

gislativa y la práctica asistencial (1931-1936). En Campos R, Villasante O and Huertas R (eds) De la «Edad de Plata al exilio» construcción y «reconstrucción» de la psiquiatría española (pp. 107-28). Madrid: Frenia.

- Ventosa Esquinaldo F. (2000). Cuidados psiquiátricos de enfermería en España (siglos XV al XX). Una aproximación histórica. Madrid: Editorial Díaz de Santos.

- Vera Pérez JA, Hernández Conesa, JM. (2011). Un análisis educativo de la formación enfermera en España durante la Segunda República. Murcia: Diego Marín Librero editor.

- Villasante, O.(2011). La Polémica en torno a los manicomios, 1916. Revista de la Asociación Española de Neuropsiquiatría, 31(112), 767-89.
- Villasante, O.(2013). Primeros intentos de profesionalización de la enfermería psiquiátrica: de la Segunda República a la posguerra española. En Simón, D, Gómez, C, Cibeira A, Villasante O (editors). Razón, locura y sociedad (pp. 315-29). Madrid: Asociación Española de Neuropsiquiatría.

- Villasante, O.(2015). El Manual del Enfermero en los Manicomios (1909) de Vicente Goyanes: preludio de la formación en enfermería psiquiátrica. Revista de la Asociación Española de Neuropsiquiatría, 35(126),401-413

- Villasante, O.(en prensa). La formación de "enfermeros psiquiátricos" durante la posguerra española: a propósito de Jaén (1939-1955). Revista Norte de Salud Mental.

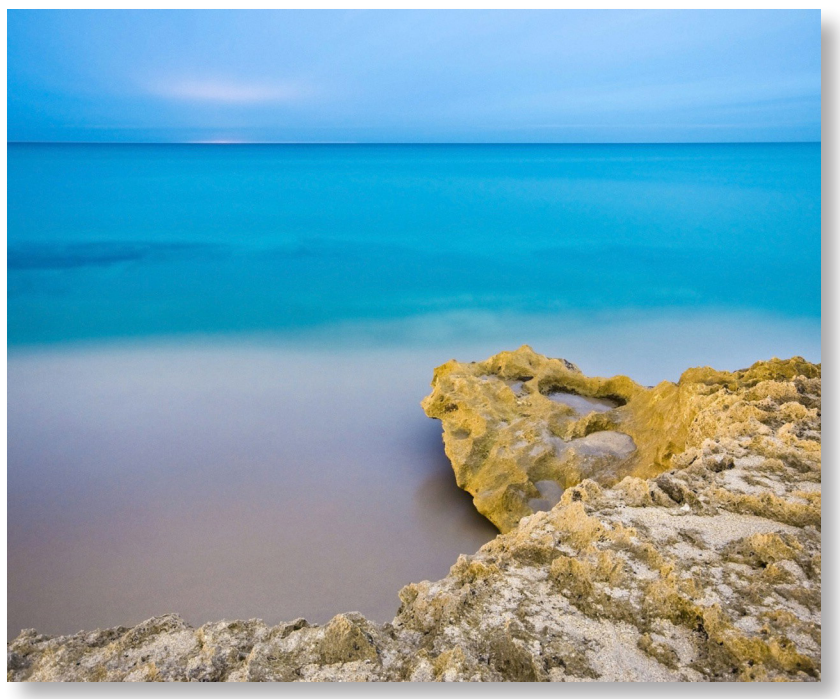

